\title{
New records of freshwater ostracods (Crustacea) from the Far East of Russia, with a checklist of recent freshwater ostracods of the region
}

\author{
Новые находки пресноводных остракод (Crustacea) \\ на Аальнем Востоке России, со списком видов современных \\ пресноводных остракод региона
}

\author{
Dmitry A. Sidorov \& Ksenya A. Semenchenko \\ А.А. Сидоров, К.А. Семенченко
}

Institute of Biology and Soil Science FEB RAS, 100-let Vladivostoku Av. 159, Vladivostok690022 Russia. E-mail: sidorov@biosoil.ru Биолого-почвенный институт ДВО РАН, пр-т 100 лет Владивостоку, 159, Владивосток 690022, Россия

KEY WORDS: Ostracoda, Cypridopsis, Ilyocypris, Metacypris, freshwater fauna, Far East.

КЛЮЧЕВЫЕ СЛОВА: Ostracoda, Cypridopsis, Ilyocypris, Metacypris, пресноводная фауна, Дальний Восток.

ABSTRACT. During surveys of the freshwater habitats in Primory and Khabarovsk provinces of the Far East of Russia, a total of 4 ostracod species were recovered and identified. Cypridopsis vidua (O.F. Müller, 1776) in the small pond in Botanical GardenInstitute FEB RAS, Primorie Province; Ilyocypris salebrosa Stepanaitys, 1960 in the Ussuri River near Kirovsky, Primorie Province; Ilyocypris dentifera Sars, 1903 in the flooded sandpit in the Bikin River basin, Khabarovsk Province and Metacypris digitiformis Smith et Hiruta, 2004 in the meander of the Nura River, Khabarovsk Province. I. dentifera and $M$. digitiformis are reported for the first time from the Far East of Russia. A species-list of recent freshwater ostracods of the Far East of Russia is provided.

РЕЗЮМЕ. Во время обследования пресноводных местообитаний в Приморском и Хабаровском краях Дальнего Востока России были обнаружены и идентифицированы 4 вида остракод. Cypridopsis vidua (O.F. Müller, 1776) в небольшом пруду в Ботаническом саду-институте ДВО РАН, Приморский край; Ilyocypris salebrosa Stepanaitys, 1960 в реке Уссури близ Кировского, Приморский край; Ilyocypris dentifera Sars, 1903 в затопленном карьере в бассейне реки Бикин, Хабаровский край и Metacypris digitiformis Smith et Hiruta, 2004 в излучине реки Нура, Хабаровский край. I. dentifera и M. digitiformis указываются впервые для фауны региона. Представлен список видов современных пресноводных остракод Дальнего Востока России.

\section{Introduction}

The freshwater ostracod fauna of the Russian Far East is extremely poorly investigated with only 21 named species previously recorded from this region in the literature (Table 1). The first records were by Bronstein [1947], who reported three species in his monograph on the freshwater ostracod fauna of Russia: Eucypris crassa (O.F. Müller, 1785) and Cypridopsis vidua (O.F. Müller, 1776) from various water bodies around Ussuryisk, and Candona krochini Bronstein, 1947 from Dalnee Lake in Kamchatka. Later, Schornikov [1986] described of a new species from Khanka Lake, Athalocythere chankensis Schornikov, 1986, and Aladin \& Schornikov [1986] reported three species from brackish and freshwaters in Primorie. Then, much later, several works devoted to different areas were published. Schornikov and Trebukhova [2001] reported new findings of eleven species from the Tumen (Tumangan) River mouth in the southwestern part of Peter the Great Bay, and Schornikov [2004] dealt with the biota of the Far-Eastern Marine Biospherical Reserve, reporting 18 species, three of them new to the region. The most recent paper, by Zenina \& Schornikov [2008], reported of the presence of Notodromas sinensis Neale et Zhao, 1991 in lower reaches of the Razdolnaya River (flowing into the Sea of Japan).

The objectives of the study were determine new records of freshwater ostracods from the Far East of Russia with compiling data on regional species list.

\section{Material and Methods}

During an expedition organized under the Amur-2 Project in 2005, several species of freshwater ostracods were collected from various freshwater habitats in the Vladivostok Botanical Garden, the Ussuri River near Kirovsky, the Bikin River basin not far from Bikin and in the Nura River basin near Gili. Sediment samples from aquatic habitats were obtained using a small hand- 


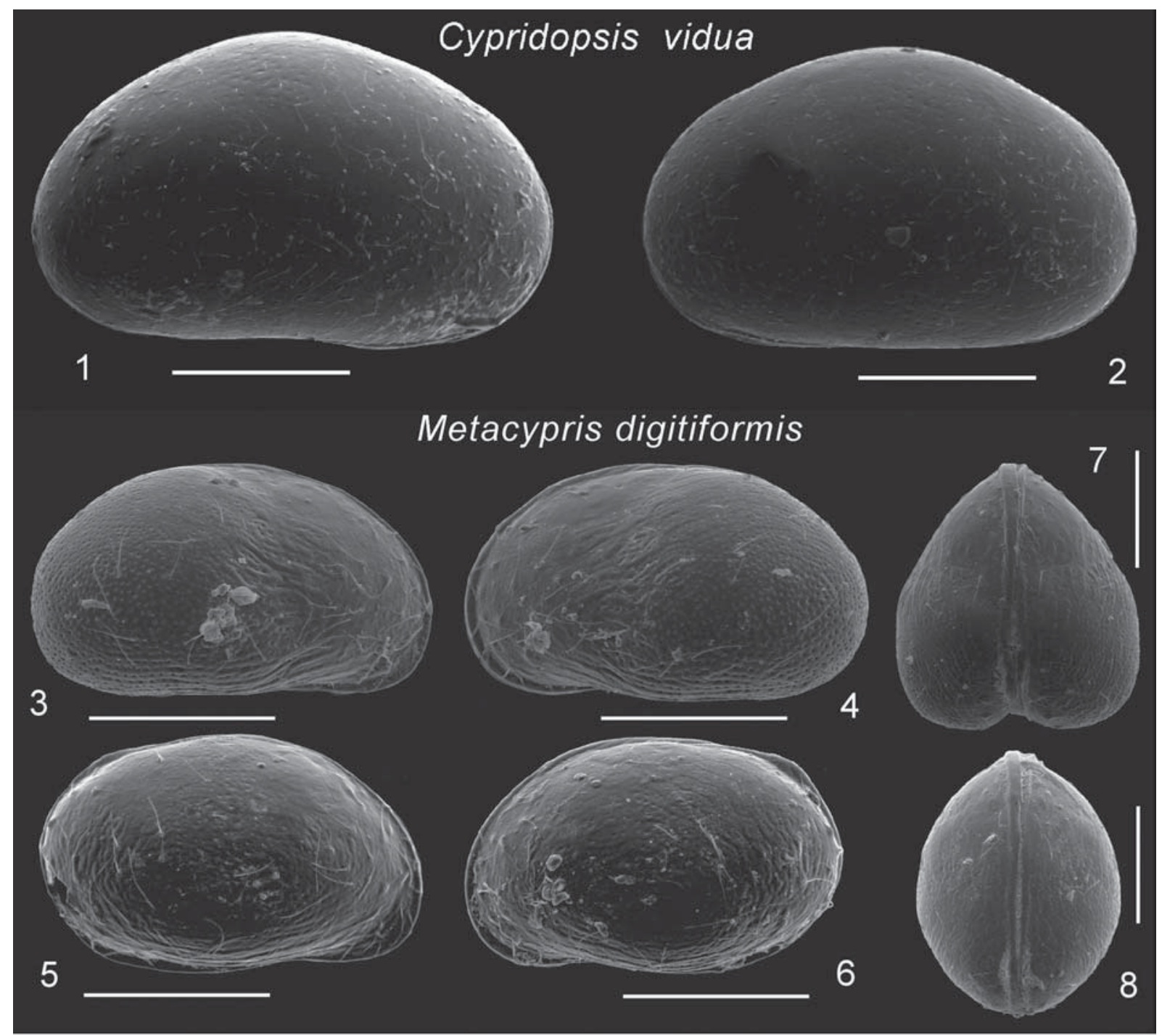

Figs 1-8. 1, 2 - Cypridopsis vidua (O.F. Müller, 1776) (X34868/Cr-1401-FESU), female, Primory Province. 1 - right valve, external view; 2 - left valve, external view. 3-8 - Metacypris digitiformis Smith et Hiruta, 2004 (X34871/Cr-1404-FESU), female (3, 4, 7), male (5, 6, 8), Khabarovsk Province. 3 - right valve, external view; 4 - left valve, external view; 5 - right valve, external view; 6 left valve, external view; 7,8 - dorsal view of carapace. Scale bars $=200 \mu \mathrm{m}$.

Рис. 1-8. 1, 2 - Cypridopsis vidua (O.F. Müller, 1776) (X34868/Cr-1401-FESU), самка, Приморье. 1 - правая створка, вид снаружи; 2 - левая створка, вид снаружи. 3-8 - Metacypris digitiformis Smith et Hiruta, 2004 (X34871/Cr-1404-FESU), самка (3, 4, 7), самец $(5,6,8)$, Хабаровский край. 3 - правая створка, вид снаружи; 4 - левая створка, вид снаружи; 5 - правая створка, вид снаружи; 6 - левая створка, вид снаружи; 7, 8 - карапакс, вид со спины. Линейки $=200 \mu \mathrm{m}$.

held dredge modified according to Schornikov [2007]. The samples were washed through a series of sieves with mesh sizes $2500 \mu \mathrm{m}$ and $250 \mu \mathrm{m}$ in the field and the resulting residues preserved in $80 \%$ alcohol solution. The samples were sorted in the lab using a dissecting microscope, Lomo MBS-9.

The ostracod specimens were placed in pure water for two-days and subsequent clearing was attained in Javelle Water (10\% sodium hypochlorite solution) by heating in a water bath. The soft parts of all recorded species were dissected and mounted on glass slides in polyvinyl lactophenol (PVL) and methylene blue staining solution. Carapaces were coated with palladium-gold before being photographed with a Carl Zeiss Evo 40 scanning electron microscope. Carapaces were stored dry in micropalaeontological cavity slides.

In the checklist information about described species according to article 11.1 of the International Code of Zoological Nomenclature [ICZN, 2004] is given only; species in "open nomenclature" are not listed. Synonymies follows by Martens \& Savatenalinton [2011]. The material examined is deposited in the Zoological Museum of the Far East State University, Vladivostok (FESU). 


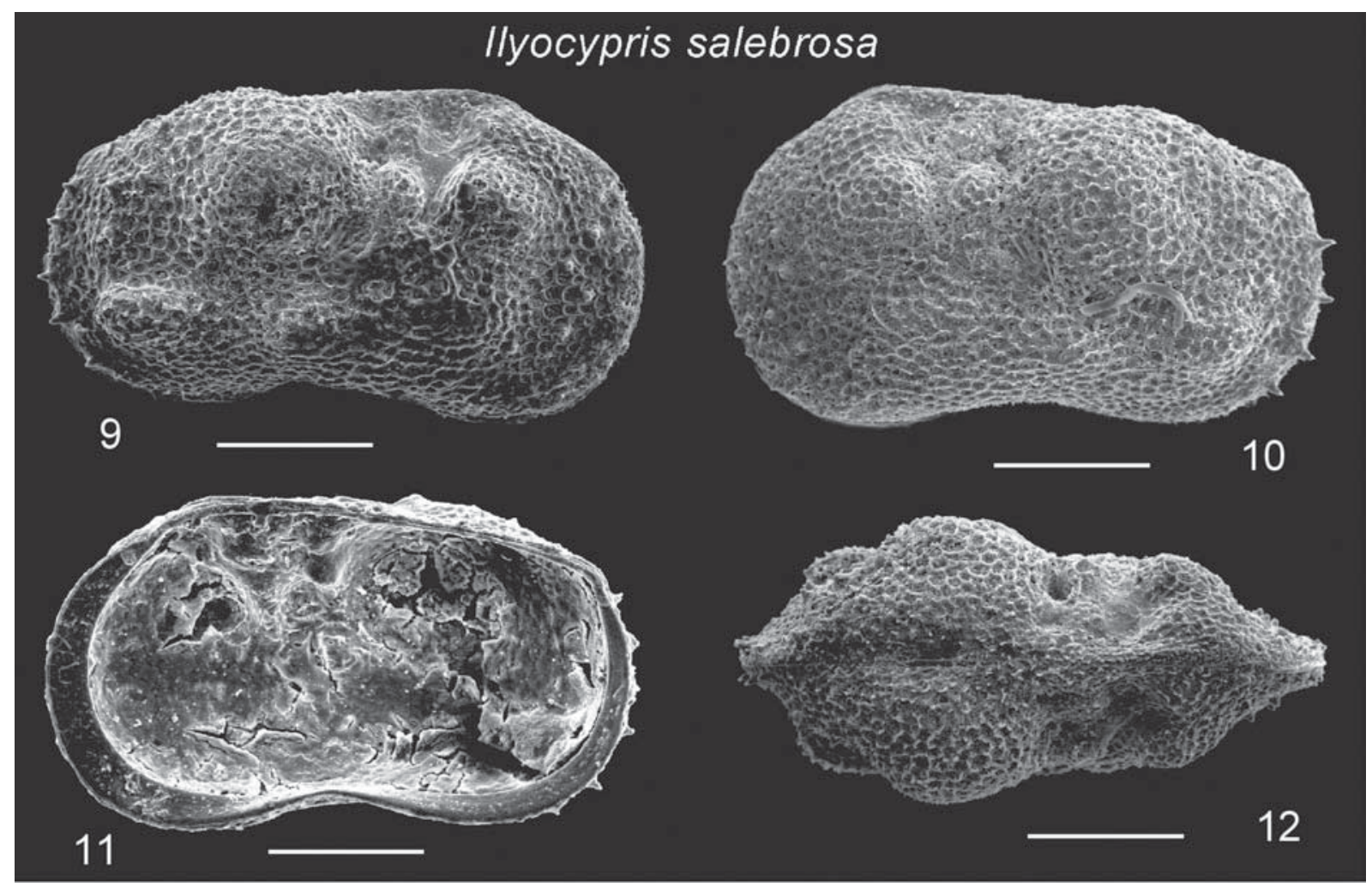

Figs 9-12. Ilyocypris salebrosa Stepanaitys, 1960 (X34869/Cr-1402-FESU), female, Primorie Province. 9 - right valve, external view; 10 - left valve, external view; 11 - right valve, internal view; 12 - dorsal view of carapace. Scale bars $=200 \mu \mathrm{m}$.

Рис. 9-12. Ilyocypris salebrosa Stepanaitys, 1960 (X34869/Cr-1402-FESU), самка, Приморье. 9 - правая створка, вид снаружи; 10 - левая створка, вид снаружи; 11 - правая створка, вид изнутри; 12 - карапакс, вид со спины. Линейки $=200 \mu \mathrm{m}$.

\section{Taxonomy}

Family Cyprididae Baird, 1845

Genus Cypridopsis Brady, 1868

Cypridopsis vidua (O.F. Müller, 1776)

Figs 1, 2.

For previous synonymies see Meisch [2000].

Cypridopsis parva G.W. Müller, 1900: Schornikov, 2004: 462. Cypridopsis vidua: Smith et al., 2011:. 9, figs 2H, 2I.

MATERIAL EXAMINED. 18 우, 4 empty valves, (X34868/ Cr-1401-FESU); Russia, Primorie Province, environs of Vladivostok, Sedanka, Botanical Garden-Institute FEB RAS, small pond, 19.10.2005 (Dudko leg.).

HABITAT. This species was collected from a small pond containing Salvinia sp.

DISTRIBUTION. C. vidua is known from Primory (Falshivy and Popova Islands, Gamova Peninsula, and Khanka Lake) and Khabarovsk regions [Schornikov, 2004]. It is a widely distributed species [Martens \& Savatenalinton, 2011].

Family Ilyocyprididae Kaufmann, 1900

Genus Ilyocypris Brady et Norman, 1889

\section{Ilyocypris salebrosa Stepanaitys, 1960}

Figs 9-12.

Ilyocypris salebrosa Stepanaitys, 1960: Schornikov, 2004: 458, fig. 1(1). - Smith et al., 2011: 17, figs 9-11.

MATERIAL EXAMINED. 3 우 (X34869/Cr-1402-FESU); Russia, Primorie Province, Kirovsky Area, near Kirovsky, Ussuri River, 45⒖741' N; 13329.598' E, 03.07.2005 (Sidorov, Semenchenko leg.).

HABITAT. This species was obtained from the bottom of the main river bed about $1.5 \mathrm{~m}$ of depth on the reach of the river. Substrate: boulders, stones, pebbles, coarse-grained sand.

DISTRIBUTION. I. salebrosa is known from Primory (Popova Island and Khanka Lake) [Schornikov, 2004]. This species has a Holarctic distribution [Martens \& Savatenalinton, 2011].

\section{Ilyocypris dentifera Sars, 1903}

Figs 13-22.

Ilyocypris dentifera Sars, 1903: Victor, Fernando, 1981: 1106, figs $1-38$.

Ilyocypris angulata Sars, 1903: Chen, 1990: 253, fig. 1.

MATERIAL EXAMINED. 28 우, $15 \mathrm{O}^{7} \mathrm{O}^{7}, 8$ juv., 10 empty valves (X34870/Cr-1403-FESU); Russia, Khabarovsk Province, Bikinsky District, flooded sandpit about $1.5 \mathrm{~km}$ from the Bikin River, $46^{\circ} 47.347^{\prime} \mathrm{N} ; 134^{\circ} 16.627^{\prime}$ E, 16.06.2005 (Sidorov, Semenchenko leg.). 


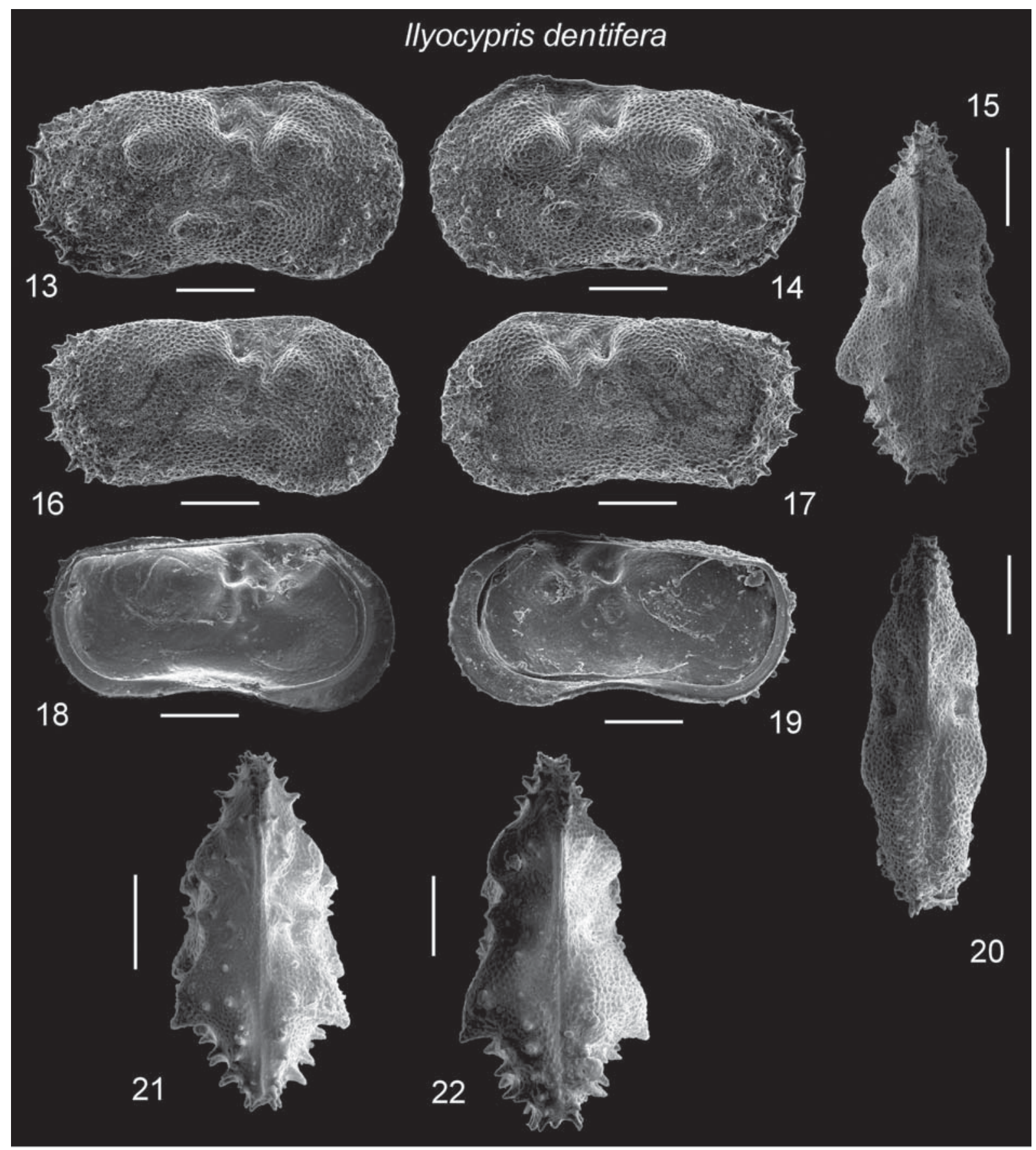

Figs 13-22. Ilyocypris dentifera Sars, 1903 (X34870/Cr-1403-FESU), female (13-15), male (16-20), juveniles (21, 22), Khabarovsk Province. 13 - right valve, external view; 14 - left valve, external view; 15 - dorsal view of carapace; 16 - right valve, external view; 17 - left valve, external view; 18 - left valve, internal view; 19 - right valve, internal view; 20-22 - dorsal view of carapace. Scale bars $=200 \mu \mathrm{m}$.

Рис. 13-22. Ilyocypris dentifera Sars, 1903 (Х34870/Cr-1403-FESU), самка (13-15), самец (16-20), личинки (21, 22), Хабаровский край. 13 - правая створка, вид снаружи; 14 - левая створка, вид снаружи; 15 - карапакс, вид со спины; 16 - правая створка, вид снаружи; 17 - левая створка, вид снаружи; 18 - левая створка, вид изнутри; 19 - правая створка, вид изнутри; 20 22 - карапакс, вид со спины. Линейки $=200 \mu \mathrm{m}$.

HABITAT. This species was collected from the bottom of a flooded sandpit about $1.3-1.5 \mathrm{~m}$ in depth. Substrate: clayey coarse-grained sand, residues of aquatic vegetation.
DISTRIBUTION. This is the first record of I. dentifera for the Far East of Russia. It is also known from Canada, Indonesia, Philippines, China and Japan [Victor \& Fernando, 1981, Yu et al., 2009]. 


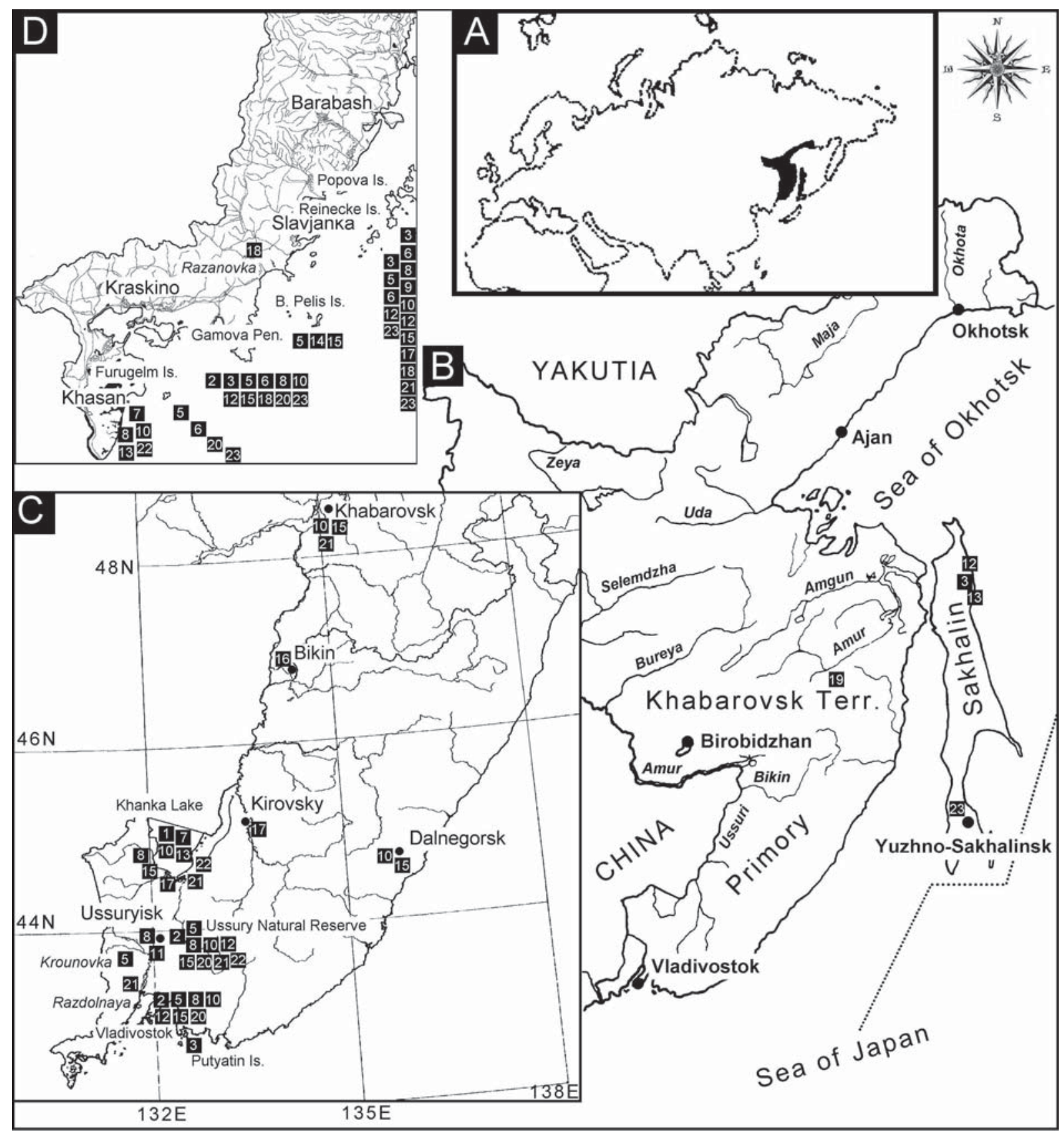

Fig. 23. Map-scheme indicating geographic distribution of Ostracoda in the Far East of Russia: A — overview of Palaearctic (Russian Far East shaded); B — enlarged Russian Far East (continental parts with Sakhalin); C — Primorie with Khabarovsk Province (south part); D - Khasan District of Primorie. Species indication as in Table 1. Source: Blank Mapping Tools, Moscow [2009].

Рис. 23. Карта-схема, отражающая географическое распространение Ostracoda на Дальнем Востоке России: А - Палеарктика, общий вид (Российский Дальний Восток заштрихован); В — Российский Дальний Восток, увеличено (континентальные части с Сахалином); C - Приморье с Хабаровским краем (южная часть); D - Хасанский район, Приморье. Обозначение видов как в Таблице 1. Источник: Коллекция бланковых карт, Москва [2009].

Family Limnocytheridae Klie, 1938

Genus Metacypris Brady et Robertson, 1870

Metacypris digitiformis Smith et Hiruta, 2004 Figs 3-8.
Metacypris digitiformis Smith et Hiruta 2004: 38, figs 2-5 (orig. descrip.).

MATERIAL EXAMINED. 13 우, $90^{7} \sigma^{7}, 5$ empty valves (X34871/Cr-1404-FESU); Russia, Khabarovsk Province, Troitzky District, $6 \mathrm{~km} \mathrm{SW}$ of Gili, meander of the Nura River, $49^{\circ} 48.485^{\prime}$ N; $137^{\circ} 07.715^{\prime}$ E, 2.07.2005 (Sidorov, Semenchenko leg.). 
Table 1. Species-list of recent freshwater Ostracoda of the Russian Far East.

Таблица 1. Список видов современных пресноводных остракод Российского Дальнего Востока.

\begin{tabular}{|c|c|c|c|c|}
\hline No. & Species & Locality & Habitat & Reference \\
\hline 1. & $\begin{array}{l}\text { Athalocythere chankensis } \\
\text { Schornikov, } 1986\end{array}$ & Primorie, Khanka Lake (NA) & lake & Schornikov [1986] \\
\hline 2. & $\begin{array}{l}\text { Bradleystrandesia reticulata } \\
\text { (Zaddach, 1844) }\end{array}$ & $\begin{array}{l}\text { Primorie, Gamova Pen. (NA), near } \\
\text { Vladivostok (NA), Ussury Natural Reserve } \\
\text { (NA), Chukchi Pen., Kolyma River and Anadir } \\
\text { River basins (NA) }\end{array}$ & pools & $\begin{array}{l}\text { Schornikov \& } \\
\text { Trebukhova [2001], } \\
\text { Schornikov [2004] }\end{array}$ \\
\hline 3. & $\begin{array}{l}\text { Candona candida (O.F. Müller, } \\
\text { 1776) }\end{array}$ & $\begin{array}{l}\text { Primorie, Gamova Pen. (NA), Popova Is. } \\
\text { (NA), Reinecke Is. (NA), Putyatin Is. (NA), } \\
\text { NE Sakhalin Is., Val River (NA) }\end{array}$ & $\begin{array}{l}\text { springs, } \\
\text { streams }\end{array}$ & $\begin{array}{l}\text { Schornikov \& } \\
\text { Trebukhova [2001], } \\
\text { Schornikov [2004] }\end{array}$ \\
\hline 4. & Candona krochini Bronstein, 1947 & Kamtchatka, Dalnee Lake (NA) & lake & Bronstein [1947] \\
\hline 5. & $\begin{array}{l}\text { Cavernocypris subterranea (Wolf, } \\
\text { 1920) }\end{array}$ & $\begin{array}{l}\text { Primorie, Gamova Pen., Spasenya Bay (NA), } \\
\text { Astafiev Bay, Gorshkov Bay (NA), Furugelm } \\
\text { Is. (NA), B. Pelis Is. (NA), Reinecke Is. (NA), } \\
\text { near Vladivostok (NA), Ussury Natural } \\
\text { Reserve (NA), Krounovka River basin, } \\
\text { Borisov plateau (NA) }\end{array}$ & $\begin{array}{l}\text { subterranean } \\
\text { waters, } \\
\text { springs }\end{array}$ & $\begin{array}{l}\text { Schornikov \& } \\
\text { Trebukhova [2001], } \\
\text { Schornikov [2004] }\end{array}$ \\
\hline 6. & Cyclocypris ovum (Jurine, 1820) & $\begin{array}{l}\text { Primorie, Gamova Pen., Astafiev Bay, } \\
\text { Gorshkov Bay (NA), Furugelm Is. (NA), } \\
\text { Reinecke Is. (NA), Popova Is. (NA) }\end{array}$ & ubiquist & $\begin{array}{l}\text { Schornikov \& } \\
\text { Trebukhova [2001], } \\
\text { Schornikov [2004] }\end{array}$ \\
\hline 7. & Cypretta seurati Gauthier, 1929 & $\begin{array}{l}\text { Primorie, Falshivy Cape (NA), near Khanka } \\
\text { Lake (NA) }\end{array}$ & $\begin{array}{l}\text { pools, rice } \\
\text { fields }\end{array}$ & $\begin{array}{l}\text { Schornikov \& } \\
\text { Trebukhova [2001], } \\
\text { Schornikov [2004] }\end{array}$ \\
\hline 8. & $\begin{array}{l}\text { *Cypridopsis vidua (O.F. Müller, } \\
1776)\end{array}$ & $\begin{array}{l}\text { Primorie, Falshivy Cape (NA), Vladivostok } \\
\text { (NA), Gamova Pen. (NA), Popova Is. (NA), } \\
\text { Ussury Natural Reserve (NA), near Ussuryisk } \\
\text { (NA), Khanka Lake (NA) }\end{array}$ & $\begin{array}{l}\text { lakes, ponds, } \\
\text { pools, } \\
\text { streams, rice } \\
\text { fields, } \\
\text { wetlands }\end{array}$ & $\begin{array}{l}\text { Bronstein [1947], } \\
\text { Aladin \& Schornikov } \\
\text { [1986], Schornikov \& } \\
\text { Trebukhova [2001], } \\
\text { Schornikov [2004], this } \\
\text { paper }\end{array}$ \\
\hline 9. & *Cyprinotus uenoi Brehm, 1936 & Primorie, Popova Is., Alekseeva Bay (NA) & $\begin{array}{l}\text { coastal } \\
\text { lagoons }\end{array}$ & Schornikov [2004] \\
\hline 10. & $\begin{array}{l}\text { *Dolerocypris fasciata (O.F. } \\
\text { Müller, 1776) }\end{array}$ & $\begin{array}{l}\text { Primorie, Falshivy Cape (NA), Gamova Pen. } \\
\text { (NA), Popova Is. (NA), near Vladivostok } \\
\text { (NA), Ussury Natural Reserve (NA), Khanka } \\
\text { Lake (NA), Pramaya Pad River basin near } \\
\text { Kamenka, Dalnegorsk (NA), near Khabarovsk } \\
\text { (NA), Chukchi Pen., Kolyma River and Anadir } \\
\text { River basins (NA) }\end{array}$ & $\begin{array}{l}\text { lakes, pools, } \\
\text { streams, rice } \\
\text { fields }\end{array}$ & $\begin{array}{l}\text { Aladin \& Schornikov } \\
\text { [1986], Schornikov \& } \\
\text { Trebukhova [2001], } \\
\text { Schornikov [2004] }\end{array}$ \\
\hline 11. & $\begin{array}{l}\text { Eucypris crassa (O.F. Müller, } \\
1785)\end{array}$ & Primorie, near Ussuryisk (NA) & ponds, pools & Bronstein [1947] \\
\hline 12. & Eucypris pigra (Fisher, 1851) & $\begin{array}{l}\text { Primorie, Gamova Pen., Gorshkov Bay (NA), } \\
\text { Furugelm Is. (NA), Reinecke Is. (NA), Popova } \\
\text { Is. (NA), near Vladivostok (NA), Ussury } \\
\text { Natural Reserve (NA) }\end{array}$ & $\begin{array}{l}\text { springs, } \\
\text { streams, } \\
\text { rarely in } \\
\text { lakes, rivers } \\
\text { and artificial } \\
\text { pools }\end{array}$ & $\begin{array}{l}\text { Schornikov \& } \\
\text { Trebukhova [2001], } \\
\text { Schornikov [2004] }\end{array}$ \\
\hline 13. & $\begin{array}{l}\text { Fabaeformiscandona subacuta } \\
\text { (Yang, 1982) }\end{array}$ & $\begin{array}{l}\text { Primorie, Falshivy Cape (NA), Khanka Lake } \\
\text { (NA), NE Sakhalin Is., Bolshoy Garomay } \\
\text { River (NA), Chaivo Bay (NA) }\end{array}$ & $\begin{array}{l}\text { wetlands, } \\
\text { lakes, pools, } \\
\text { coastal } \\
\text { lagoons }\end{array}$ & $\begin{array}{l}\text { Schornikov \& } \\
\text { Trebukhova [2001], } \\
\text { Schornikov [2004] }\end{array}$ \\
\hline 14. & $\begin{array}{l}\text { Heterocypris barbara (Gauthier et } \\
\text { Brehm, 1928) }\end{array}$ & Primorie, B. Pelis Is. (NA) & $\begin{array}{l}\text { lakes, } \\
\text { synanthropic } \\
\text { pools }\end{array}$ & Schornikov [2004] \\
\hline 15 . & $\begin{array}{l}\text { *Heterocypris incongruens } \\
\text { (Ramdohr, 1808) }\end{array}$ & $\begin{array}{l}\text { Primorie, Gamova Pen. (NA), B. Pelis Is. } \\
\text { (NA), Popova Is. (NA), Vladivostok (NA), } \\
\text { Ussury Natural Reserve (NA), Khanka Lake } \\
\text { (NA), Pramaya Pad River basin near Kamenka, } \\
\text { Dalnegorsk (NA), near Khabarovsk (NA) }\end{array}$ & $\begin{array}{l}\text { lakes, } \\
\text { synanthropic } \\
\text { pools }\end{array}$ & $\begin{array}{l}\text { Aladin \& Schornikov } \\
\text { [1986], Schornikov \& } \\
\text { Trebukhova [2001], } \\
\text { Schornikov [2004] }\end{array}$ \\
\hline 16. & Ilyocypris dentifera Sars, 1903 & $\begin{array}{l}\text { Khabarovsk, Bikin River basin }\left(46^{\circ} 47.347^{\prime} \mathrm{N} \text {; }\right. \\
\left.134^{\circ} 16.627^{\prime} \mathrm{E}\right)\end{array}$ & $\begin{array}{l}\text { artificial } \\
\text { pools }\end{array}$ & this paper \\
\hline 17. & $\begin{array}{l}\text { *Ilyocypris salebrosa Stepanaitys, } \\
1960\end{array}$ & $\begin{array}{l}\text { Primorie, Popova Is. (NA), Khanka Lake } \\
\text { (NA), Ussuri River }\left(45^{\circ} 15.741^{\prime} \mathrm{N} \text {; }\right. \\
\left.133^{\circ} 29.598^{\prime} \mathrm{E}\right)\end{array}$ & $\begin{array}{l}\text { coastal } \\
\text { lagoons, } \\
\text { lakes, } \\
\text { streams }\end{array}$ & $\begin{array}{l}\text { Schornikov [2004], this } \\
\text { paper }\end{array}$ \\
\hline 18. & $\begin{array}{l}\text { *Limnocythere stationis Vávra, } \\
1891\end{array}$ & $\begin{array}{l}\text { Primorie, Gamova Pen. (NA), Popova Is. } \\
\text { (NA), Razanovka River basin, Khasan (NA), } \\
\text { Kamtchatka (NA) }\end{array}$ & $\begin{array}{l}\text { lakes, pools, } \\
\text { rice fields, } \\
\text { rarely in } \\
\text { streams }\end{array}$ & $\begin{array}{l}\text { Aladin \& Schornikov } \\
\text { [1986], Schornikov \& } \\
\text { Trebukhova [2001], } \\
\text { Schornikov [2004] }\end{array}$ \\
\hline
\end{tabular}


Table 1 (contituing)

Таблица 1 (продолжение)

\begin{tabular}{|l|l|l|l|l|}
\hline 19. & $\begin{array}{l}\text { Metacypris digitiformis Smith et } \\
\text { Hiruta, 2004 }\end{array}$ & $\begin{array}{l}\text { Khabarovsk, Nura River basin (4948.485' N; } \\
137^{\circ} 07.715^{\prime} \text { E) }\end{array}$ & wetlands & this paper \\
\hline 20. & Nannocandona faba Ekman, 1914 & $\begin{array}{l}\text { Primorie, Gamova Pen., Astafiev Bay (NA), } \\
\text { Furugelm Is. (NA), Vladivostok (NA), Ussury } \\
\text { Natural Reserve (NA), South Kuril Isl. (NA), } \\
\text { Kamtchatka (NA) }\end{array}$ & $\begin{array}{l}\text { springs, } \\
\text { seeps }\end{array}$ & $\begin{array}{l}\text { Schornikov \& } \\
\text { Trebukhova [2001], } \\
\text { Schornikov [2004] }\end{array}$ \\
\hline 21. & $\begin{array}{l}\text { Notodromas sinensis Neale et Zhao, } \\
1991\end{array}$ & $\begin{array}{l}\text { Primorie, Popova Is. (NA), Ussury Natural } \\
\text { Reserve (NA), Razdolnaya River (NA), } \\
\text { Khanka Lake (NA), near Khabarovsk (NA) }\end{array}$ & $\begin{array}{l}\text { lakes, } \\
\text { pools }\end{array}$ & $\begin{array}{l}\text { Schornikov \& } \\
\text { Trebukhova [2001], } \\
\text { Schornikov [2004], } \\
\text { Zenina \& Schornikov } \\
\text { [2008] }\end{array}$ \\
\hline 22. & $\begin{array}{l}\text { Physocypria kraepelini G.W. } \\
\text { Müller, 1903 }\end{array}$ & $\begin{array}{l}\text { Primorie, Falshivy Cape (NA), Ussury Natural } \\
\text { Reserve (NA), Khanka Lake (NA) }\end{array}$ & $\begin{array}{l}\text { lakes, } \\
\text { streams, } \\
\text { wetlands }\end{array}$ & $\begin{array}{l}\text { Schornikov \& } \\
\text { Trebukhova [2001], } \\
\text { Schornikov [2004] }\end{array}$ \\
\hline 23. & $\begin{array}{l}\text { Scottia birigida } \text { Smith et al., 2002 } \\
\text { springs, } \\
\text { seeps }\end{array}$ & $\begin{array}{l}\text { Schornikov \& } \\
\text { Trebukhova [2001], } \\
\text { Schornikov [2004] }\end{array}$ \\
\hline
\end{tabular}

Notes: * - sometimes dwells in brackish waters, NA — coordinates not available.

HABITAT. This species was obtained from the bottom of a swampy oxbow lake at a depth of about 1.2 $\mathrm{m}$. Substrate: black silt, decaying plant remains, detritus, aquatic vegetation, submerged logs, tussocks.

DISTRIBUTION. Japan [Smith \& Hiruta, 2004].

REMARKS. Previously by Schornikov \& Trebukhova [2001], and Schornikov [2004] reported several findings in Primory of the similar species Metacypris hebeiensis Huang, 1985, which was described from the Pleistocene deposits of Hebei, China [Huang, 1985].

\section{Results}

Including the two new records presented herein, a total of 23 species of freshwater ostracods have been identified from the region (Fig. 23). A number of previous publications have listed species in "open nomenclature", without proper descriptions and only with information on distribution and ecology. Undescribed taxa are not included in the present species-list (see Table 1), but it should be noted that several other genera, Vestalenula Rossetti et Martens, Cryptocandona Kaufmann, Pseudocandona Kaufmann and Cypria Zenker, have been reported from the Primory by Schornikov \& Trebukhova [2001], and Schornikov [2004]. However, no named species of these genera have been so far reported from the region [Schornikov, 1995].

Ilyocypris japonica Okubo, 1990, seems to be closely related to the undescribed species Ilyocypris sp. 1 . sensu Schornikov \& Trebukhova [2001] [Schornikov, 2004, p. 458, 464, fig. 1, 2]; they share similarities in size ranges and the sculpture of the shell. The ostracod fauna of the Russian Far East includes Palearctic, Holarctic, and one endemic species (Athalocythere chankensis). No doubt more thorough investigations will result in more discoveries of this poorly known fauna in the region.
ACKNOWLEDGEMENTS. We are thankful to Dr. Smith (Lake Biwa Museum, Shiga) for helpful suggestions that greatly improved the paper and for revising the English of manuscript. We would also like to thank Dr. Yamada (University of Tokyo) for placing at our disposal SEM images of I. japonica and Mr. Fomin, expert at the Centre for Public Use (Institute of Marin Biology, Vladivostok). We would also like to thank anonymous reviewers for critically reading and commenting on the manuscript.

This study was supported by the Presidium of the Far Eastern Branch of the Russian Academy of Science under the research program "Amur-2" and \# 12-I-П30-01.

\section{References}

Aladin N.V., Schornikov E.I. 1986. [Salinity adaptations and osmoregulatory abilities in the Ostracoda from the Sea of Japan. Part 1] // Zoologichesky Zhurnal. Vol.65. P.829-836 [in Russian].

Bronstein Z.S. 1947. [Ostracoda of fresh waters] // Fauna SSSR. Rakoobraznye. T.2. No.1. Moscow-Leningrad: AN SSSR Publ. 371 p. [in Russian]

Chen S. 1990. [Description of a male Ilyocypris angulata Sars (Crustacea: Ostracoda)] // Acta Micropalaeontologica Sinica. Vol.7. P.253-256 [in Chinese].

Huang B. 1985. [Ostracoda and their geological significance in the Pleistocene strata of lower reaches of Sanggan River] // Monographs of Nanjing Institute of Geology and Paleontology Chinese Academy of Sciences. No.21. P.85-107 [in Chinese].

Martens K., Savatenalinton S. 2011. A subjective checklist of the Recent, free-living, non-marine Ostracoda (Crustacea) // Zootaxa. Vol.2855. P.1-79.

Meisch C. 2000. Freshwater Ostracoda of western and central Europe // Schwoerbel J., Zwick P. (eds.). Süßwasserfauna von Mitteleuropa. Heidelberg: Spektrum Akademischer Verlag. $522 \mathrm{p}$.

Schornikov E.I. 1986. [The new taxa of the ostracode Limnocytheridae from Far-Eastern and Central Asian water bodies] // Levanidova I.M. (ed.). Donnye organizmy presnykh vod Dalnego Vostoka. Vladivostok: Dal'nevostochnoe otdelenie AN SSSR. P.19-29 [in Russian].

Schornikov E.I. 1995. Subclass Ostracoda. Hydrobiological investigations in Ussuri Nature Reserve. Part $1 / /$ Vshivkova T.S. 
(ed.). Freshwater fauna (biodiversity and biographical analysis). Vladivostok: Dalnauka. P.18-19.

Schornikov E.I. 2004. Crustacea: Ostracoda // Tyurin A.N., Drozdov A.L. (eds.). Far-Eastern Marine Biospherical Reserve. Biota. Vladivostok: Dalnauka. P.458-465.

Schornikov E.I. 2007. Recent and fossil (Quaternary) ostracods of the Tien Shan mountain lakes bassins Sonkul Lake and Chatyrkul Lake // Romanovsky, V.V. (ed.). Climate, glaciers, lakes of the Tien Shan: journey to the past. Bishkek: Ilim. P.110-166.

Schornikov (Shornikov) E.I., Trebukhova Yu.A. 2001. Ostracods of brackish and fresh waters of southwestern coast of Peter the Great Bay // Kasyanov V.L., Vaschenko M.A., Pitruk D.L. (eds.). The state of the environment and biota of the southwestern part of Peter the Great Bay and the Tumen River mouth. Vladivostok: Dalnauka. P.56-84.

Smith R.J., Hiruta S. 2004. A new species of Metacypris (Limnocytherinae, Cytheroidea, Ostracoda, Crustacea) from Hokkaido, Japan // Species Diversity. Vol.9. P.37-46.
Smith R.J., Janz H., Okubo I. 2011. Recent Cyprididae and Ilyocyprididae (Crustacea: Ostracoda) from Lake Biwa, Japan, including a summary of the lake's ostracod fauna // Zootaxa. Vol.2874. P.1-37.

Victor R., Fernando C.H. 1981. Ilyocypris dentifera Sars, 1903 versus Ilyocypris angulata Sars, 1903. A taxonomic study of two species of Ilyocypris Brady and Norman, 1889 (Crustacea, Ostracoda) described from China // Canadian Journal of Zoology. Vol.59. P.1103-1110.

Yu N., Zhao Q., Li E., Chen S., Chen L. 2009. An updated and annotated checklist of recent nonmarine ostracods from China // Zootaxa. Vol.2067. P.29-50.

Zenina M.A., Schornikov E.I. 2008. Ostracod assemblages of the freshened part of Amursky Bay and lower reaches of the Razdolnaya River (Sea of Japan) // Lutaenko K.A., Vaschenko M.A. (eds.). Ecological studies and the state of the ecosystem of Amursky Bay and the estuarine zone of the Razdolnaya River (Sea of Japan). Vladivostok: Dalnauka. P.156-185.

Responsible editor V.A. Spiridonov 